\title{
Survey on Multi-Party Privacy Conflicts in Social Media
}

\author{
R. Aravinth ${ }^{1}$, G. Bharath ${ }^{2}$, J. Dinesh ${ }^{3}$, M. Hari ${ }^{4}$, N. Kathirvel ${ }^{5}$ \\ UG, Department of CSE, MKCE, Karur, India ${ }^{1,2,3,4,5}$
}

\begin{abstract}
On-line Social Networks (OSNs) expend knowing commanding movement in clean years and develop into portal in wish of jillions of net quit clients. Those provider deal alluring methodology for digitals organize contact and truths dissemination, however to boot trade a numeral of wellbeing and secrecy issues. While OSNs concur clients to control get right of passage to pooled measurements, they once don't convey any gadget to instructor echoer information associated with a modest bunch of customers. To the present outstanding degrees, we tend to supporter connect come back with reference to change the fortress of shared measurements identified with numerous handlers in OSNs. We tend to put along a privilege of get to controller model to seizure the see of helpful quit horsemen objectives, on board through approach of a multiparty strategy measurement test and a way organization invention. Also, we tend to contemporaneous a judgment showing of our affirmation system original that faces United States to control the geologies of winning sensibility solvers to finish interminable investigation family commitments on our prototype. We tend to as pleasantly articulate a confirmation of-thought organization of our meld as piece of a sales in Face digital book and convey ease of use task and refinement estimation of our gadget. Contraptions common completed system Broadcasting isn't introductory rate with more distant than single customer privacy — e.g., picks that paint several clients, editorials that remark complex client, happenings amid which few client is asked for then on.
\end{abstract}

Keywords: Dissemination, Geologies, Contemporaneous, Contraptions.

\section{INTRODUCTION}

Colleague delivering is potential in non-home grown cleverness that advances information built up structures. Such arrangements are centralized server PC educational modules that comprehend immense totals of capacity, documentations and highbrow gadgets to convey answers for reasonable glitches. A gathered works breakdown is a confirmation of what has been reachable on a point by method for prepared researchers and colleagues. Discontinuously you'll be addressed to scribble down one as a separated obligation, yet more prominent as often as possible its miles extent of the initiation to thesis, watch record, or estimation. In composing the true to life audit, your reason is to move in your booklover what truths and contemplations were initiate on a business undertaking, and what their benefit and weakness are. As a bit of engraving, the writing evaluation should be portrayed with the guide of an authoritative it isn't generally essentially a clarifying rundown of the quantifiable accessible, or a gathering of abstracts. Other than expanding your knowhow around the issue, composing an announcement investigation gives you a chance to engender and set up abilities in limits

1. INFORMATION SEEKING: Special mind to: the ability to examination the writings creatively, the usage of manual or convenient PC capacities, to inventory an intense and brisk of valuable articles and reports

2. CRITICAL APPRAISAL: The degree to spread methods of insight of examination to reason cool peered toward and legitimate grants.

\section{DOMAIN INTRODUCTION}

Understanding portrayal and mental component is that the a piece of non-home grown insightfulness gung hot to in capacity of datum sound the part in an extremely game plan that a data preparing framework gadget will work to clarify multipart obligations like make a forecast a smooth-going or having a voice correspondence in an exceptionally common annals. Family station joins restrictions from acknowledgment in regards to however people handle ontogeny issues and largesse information in summon to association formalisms with the aim to change shifted frameworks quiet to point and constitution. Insight showing and discerning conjointly coordinates results from sensibility to motorization in a few classes of cerebral, similar to the utility of rulebooks or the hover of relative's members of sets and subcategories.

\section{III.LITERATURE SURVEY}

Multi-Party Risk Threats in Social Networks"

Thomas K, Nicola M, and Grier C.As the wonder of interpersonal organizations develops, the figures individual's show bureau to the unhindered has theoretically risk for discrete security [1]. While social setups befits clients constrain push forward to their particular insights, there's crisply no hardware to authorize security troubles over glad transferred with the assistance of different controllers [2]. As association duplicates and lies are joint by abuse partners and family, individual assurance goes outside the decision of what an individual transfers around himself and creates perception of what 
each system part reveals. On this paper, we investigate how the deficiency of joint privateer's controls over substance can continually screen ordered data around a man which incorporates decisions, connections, dialogs, and pictures. Altogether, we screen Face digital book to find scenes wherein contrasting protection settings between mates will screen records that no less than one purchaser assumed keep on being private. With the guide of check the data exhibited on this form, we give a clarification to how a man's private propensities might be gathered from as a general rule being recorded as a pal or expressed in a story[3]. To facilitate this possibility, we indicate how Face digital book's privateer's adaptation can be custom fitted to ingrain multi-festivity protection. "A Survey of Privacy in Multi-Agent Systems "J. M. Such, A. Espinosa. Privateers has been a trouble for human extensive before the unsafe increment of the web[4].

The advances in measurements capacities have additionally amplified these issues. this is on account of the expanding power and issue of portable PC programming gives each first class potential outcomes for people, however likewise colossal dangers to private privateers. Autonomous merchants and Multi-operator structures are case of the level of issue of PC projects. Self-sufficient operators for the most part gauze private fabric depicting their doyens, and in this way they play a urgent position in an antibacterial privateers. besides, sovereign retailers themselves is likewise used to increase the mystery of convenient PC applications through captivating addition of the basic scenes they give, and also reproduction cunning, master movement, freedom, and alike. This article acquaints the matter of medication isolation in portable workstation bundles and its individual from the family to self-decision retailers and Multioperator invention. It conjointly examinations security related headings at interims the universe of Multi-operator structures and pinpoints open challenges to be discussion over with by abuse imminent investigation[5]. Collaborative Access Control in Online Social Networks" B. Carminative and E. Ferrari[6]. Topology principally based induction administration is nowadays a typical for cautious belonging in on-line Social Networks (OSNs) each inside the examination organize and attractive OSNs. to keep with this model, underwriting regions require the cooperation's and perchance their force and certainty lustrous that must be constrained to unfold between the requestor and furthermore the proposes that titleholder to make the premier one to get to the mandatory give. Amid this broadside, we have a tendency to choose however topology-basically fundamentally based get admission to system is likewise increased through abusing the relationship among OSN clients that will be that the substance of any OSN. The stipulation of client joint effort all through inspire admission to oversee bearing emerges by recommends that of reality that, unmistakable from oldschool settings, in most OSN administrations clients will situation diverse clients in property, and therefore it's normally not possible for a person to direct the financial backing uncovered by further individual. For this reason, we have a tendency to present helpful duty strategies, get right of section to senator standards for choosing an extreme and brisk of joint clients that request be refined all through get right of passage to manage organization.

Besides, we tend to show however customer connection might be hangdog for arrangement creation and that we blessing expanding on help of joint scope execution. A Tool for Eliciting Tie Strength and User Communities in Social Networking Services"R. L. Fugue, J. M. Such, A. Espinosa, And A. Garcia-Forms[8] The practice of long range informal communication offices in conjunction with Facebook has dangerously individual at interims the past couple of years. Clients see these SNSs as proper instrumentality to discover families and interrelate with them. Besides, SNS permit their clients to extent snaps, videocassettes, and set off their considerations and states of mind. In any case, clients are regularly aggravated generally their isolation while misuse SNSs. that is inferable from the very truth the community analogies of a subject is additionally manufactured through depictions or perceptions sent on an informal organization. On this way, current examination confirm that clients are nervewracking higher instruments to secure their privateers. The appropriate guess for cure this can be a privateer's colleague programming framework operator that continually proposes a privateers scope for anything to be shared on a SNS. The essential stride for developing such partner operator which can evoke imperative learning that may bring about right privateers scope forecasts. especially, the information wished is individual groups and furthermore the force of clients' allure, which, as directed by approach of current experimental verification, are the most extreme critical elements that drive discourse act in SNSs. we have a tendency to boot blessing partner exploratory investigation identifying with thirty eight subjects that affirmed that BFF will prominently lighten the heap of vexing groups and offer power. [7]"Mapping User Preference to Privacy Default Settings. R. Lip Ford, and A. Besmear Managing the security of on-line records is additionally an intense venture much of the time returning into the setup of a scope of spots for example, Face digital book clients control that observers have get right of passage to their report information and submit, however companions will interconnect with them through class, and furthermore the approach others will scrounge around for the and extra secrecy commitments[9]. By and large, the avoidance wellbeing settings are liberal and appear to be intended to offer information apportioning assemble than security. Utilize granular insurance settings. On this paper, we're examining regardless of whether or not default insurance settings on informal community sites is a ton of bespoken to the choices of customers. We have a tendency to anticipate clients' insurance states of mind and circulating choices for ordinary SNS information objects. We have a tendency to investigate the sound of grouped plans, exhaustive of dissecting regardless of whether privateer's outlook division is wont to build 
default settings. Our belongings educate that the use concerning target showcase portrayals from system records to make default privateers settings will higher sound clients' most famous privateers settings[10].

\section{EXISTING SYSTEM}

Web-based social networking are had by means of more than one administrators, the client that transfers the thing is permissible to line its privateers areas (i.e., WHO will get admission to the question). that is a magnificent and insightful drawback as individual's secrecy top picks for co-claimed devices generally fighting, therefore spreading the choices of least complex one festival dangers such contraptions being imparted to undesirable beneficiaries, that may prompt to wellbeing infringement with outrageous results (e.g., clients losing their employments, being digital pediculate, and so on tests of devices grasp pictures that depict a modest bunch of guardians, input that reference more than one clients, motivation amid which complex staff are asked, and so forth. Multi-party privateer's foundation is, in this way, of fundamental rank for supporters to fittingly reservation their classification in Social Media. Current confirmation clients genuine ordinarily offer cooperatively to get relate settlement on classification settings for co-claimed learning in Social Media. Mostly, clients are respected to be generally open house distinctive client's inclinations, and that they are needing to insignia two or three establishments to acknowledge assertion unexpected the exact country.

\section{DISADVANTAGES}

> Social Media have a course on particular individual's privations things shared by approach of themselves Unreliable and inaccurate results

$>\quad$ The not have of multi-festivity space to yourself organization encourage in popular focal point of-the-road.

$>$ Social Media foundations collect clients not successful to decidedly administration to whom those bits and parts are really spread or not

\section{PROPOSED SYSTEM}

On one needle, dispose of a tag since a photo can best spare you diverse disciples from seeing a man's shape by method for the suggestion interface, yet the client's two occasions remains oversaw inside the depiction. Since the valid gain passage to power rules can't be changed, the purchaser's photograph remains to be discovered to every single authorized client. On the other hand, answering to OSNs best permits us to both keep up and erase the substance material. Any such double choice from OSN supervisor is either too free or excessively banishing, depending on the OSN's supervision and requiring various people to portrayal their call for on a similar stuffing. Subsequently, it is imperative to amass a valuable and flexible inspire admission to compose component for OSNs, obliging the uncommon authorization necessities originating from various related clients for supervision the joint certainties cooperatively. Exceptionally shining related composition proposed instruments to decide multibirthday party privations war in online networking. Some of them need too considerably human obstruction over the span of the contentions determination strategy, by utilizing obliging clients to clear up the contentions physically or near physically.

\section{EXPERIMENTAL RESULT}

\author{
Modules: \\ $>$ Individual Privacy \\ $>$ Conflict detection \\ $>$ Estimating the willingness \\ Conflict resolution
}

We think a one response for community control of shared insights in OSNs. A MPAC adaptation get to be distinctly detailed, together with a multiparty scope prerequisite plan and like that arrangement evaluation component. Advance, we've included an approach for speaking to and thinking about our ponder adaptation. This has the maturing to decrease the amount of guide individual intercessions to play out a legitimate response for all festival stressed in multi-festivity privations clashes. moreover, the becoming more acquainted with likewise affirmed the remuneration that a versatile component, we close by on this paper can offer with expense to additional static methodologies of amassing clients' man or lady privations inclinations, which are not proficient to advance to unique circumstances and have been extraordinary from what the clients did themselves.

\section{REFERENCES}

[1] K. Thomas, C. Grier, and D. M. Nicol, "unfriendly: Multi-party privacy risks in social networks," in Privacy Enhancing Technologies. Springer, 2010, pp. 236-252.

[2] J. M. Such, A. Espinosa, and A. Garc'1a-Fornes, "A survey of privacy in multi-agent systems," The Knowledge Engineering Review, vol. 29, no. 03, pp. 314-344, 2014.

[3] R. Wishart, D. Corapi, S. Marinovic, and M. Sloman, "Collaborative privacy policy authoring in a social networking context," in POLICY. IEEE, 2010, pp. 1-8.

[4] R. L. Fogu'es, J. M. Such, A. Espinosa, and A. Garcia-Fornes, "BFF: A tool for eliciting tie strength and user communities in social networking services," Information Systems Frontiers, vol. 16, no. 2, pp. 225-237, 2014.

[5] J. Watson, H. R. Lipford, and A. Besmer, "Mapping user preference to privacy default settings," ACM Transactions on ComputerHuman Interaction (TOCHI), vol. 22, no. 6, p. 32, 2015.

[6] Lampinen, V. Lehtinen, A. Lehmuskallio, and S. Tamminen, "We're in it together: interpersonal management of disclosure in social network services," in Proc. CHI. ACM, 2011, pp. 3217-3226.

[7] P.Wisniewski, H. Lipford, and D.Wilson, "Fighting for my space: Coping mechanisms for sns boundary regulation," in Proc. CHI. ACM, 2012, pp. 609-618.

[8] Besmer and H. Richter Lipford, "Moving beyond untagging: photo privacy in a tagged world," in ACM CHI, 2010, pp. 1563- 1572.

[9] S Saravanan, VVenkatachalam, S Then Malligai"Optimization of SLA violation in cloud computing using artificial bee colony"2015, 1(3), 323 -327 ISSN: 2394-9260, pp:410-414.

[10] S Saravanan, V Venkatachalam ,"Advance Map Reduce Task Scheduling algorithm using mobile cloud multimedia services architecture" IEEE Digital Explore,pp21-25,2014. 